\title{
Precise DNA Cloning via PAMless CRISPR-SpRYgests
}

\section{Authors:}

Kathleen A. Christie ${ }^{1-3}$, Jimmy A. Guo ${ }^{1,2,4}$, Rachel A. Silverstein ${ }^{1,2,4}$, Roman M. Doll ${ }^{1,2,5}$, Megumu Mabuchi $^{6}$, Hannah E. Stutzman ${ }^{1,2}$, Linyuan $\mathrm{Ma}^{1-3}$, G. Brett Robb ${ }^{6}$, Benjamin P. Kleinstiver ${ }^{1-3,{ }^{*}}$

\section{Affiliations:}

${ }^{1}$ Center for Genomic Medicine, Massachusetts General Hospital, Boston, MA, 02114, USA

2 Department of Pathology, Massachusetts General Hospital, Boston, MA, 02114, USA

${ }^{3}$ Department of Pathology, Harvard Medical School, Boston, MA, 02115, USA

${ }^{4}$ Biological and Biomedical Sciences Program, Harvard University, Boston, MA, 02115, USA

${ }^{5}$ Molecular Biosciences/Cancer Biology Program, Heidelberg University, and German Cancer Research Center, DKFZ, Heidelberg, Germany

${ }^{6}$ New England Biolabs, Ipswich, MA, 01938, USA

*Correspondence to: bkleinstiver@mgh.harvard.edu

\section{Abstract:}

While restriction enzymes (REs) remain the gold-standard for manipulating DNA in vitro, they have notable drawbacks including a dependence on short binding motifs that constrain their ability to cleave DNA substrates. Here we overcome limitations of REs by developing an optimized molecular workflow that leverages the PAMless nature of a CRISPR-Cas enzyme named SpRY to cleave DNA at practically any sequence. Using SpRY for DNA digests (SpRYgests), we establish a method that permits the efficient cleavage of DNA substrates at any base pair. We demonstrate the effectiveness of SpRYgests using more than 130 gRNAs, illustrating the versatility of this approach to improve the precision of and simplify several cloning workflows, including those not possible with REs. We also optimize a rapid and simple one-pot gRNA synthesis protocol, which reduces cost and makes the overall SpRYgest workflow comparable to that of RE digests. Together, SpRYgests are straightforward to implement and can be utilized to improve a variety of DNA engineering applications. 


\section{Body:}

Restriction enzymes (REs) transformed the field of molecular cloning by enabling and accelerating the assembly of recombinant DNA fragments ${ }^{1}$. REs commonly utilized for cloning applications recognize sequence motifs typically 4-8 base pairs in length and generate DNA double strand breaks (DSB) (Fig. 1a). Despite a diverse catalog of REs, there remain challenges for molecular cloning since the availability of these motifs in DNA substrates is stochastic, and to be useful, the RE motif should be conveniently located and generally must only occur once (so-called 'single cutters'). Aside from purposefully designed multiple-cloning sites (MCSs) harboring several unique RE motifs (Sup. Fig. 1a), most RE motifs either do not occur in a substrate or are found more than once, preventing specific digestion of a DNA substrate to generate the desired fragments. Given these caveats, most laboratories purchase a repertoire of REs which can be costly and is incomplete since REs cannot comprehensively address all sequences.

CRISPR-Cas nucleases are one potential alternative platform that can cleave DNA in a customizable manner. Most of the DNA-targeting specificity of Cas enzymes is provided by a guide RNA (gRNA) that can be programmed to bind to nearly any sequence ${ }^{2}$ (Sup. Fig. 1b). However, canonical Cas 9 nucleases are also encumbered by the requirement to recognize a short DNA motif adjacent to the target site, known as the protospacer adjacent motif ${ }^{3}$ (PAM; Sup. Fig. 1b). The dependence on the availability of a PAM proximal to the target site prohibits precise cleavage of DNA substrates (Sup. Fig. 1c), including for in vitro DNA digests ${ }^{4}$. To overcome this limitation, a nearly PAMless CRISPR-Cas variant named SpRY was recently engineered ${ }^{5}$ that can target DNA sites with NNN PAMs in human cells, with a preference for NRN PAMs over NYN PAMs (where $R$ is $A$ or $G$ and $Y$ is $C$ or $T$; Fig. 1a and Sup. Fig. 1d). Given that SpRY is no longer dependent on a PAM, we investigated whether SpRY could act as a fully programmable DNA endonuclease to cleave at any DNA base in vitro, vastly simplifying the design and execution of molecular cloning protocols.

We first investigated whether SpRY could generate DSBs along a DNA substrate at various locations harboring different PAMs. In our initial assays (Fig. 1b), we performed in vitro digests utilizing overexpressed SpRY protein from human cell lysates (Sup. Figs. $2 a$ and 2 b) along with gRNAs produced using optimized and rapid in vitro transcription (IVT) conditions (Sup Figs. 3a-3c and Sup. Note 1). We assessed SpRY activity in vitro by performing SpRY DNA digests (SpRYgests) against 20 different target sites sampling NRN and NYN PAMs across a linearized plasmid substrate (Figs. 1c and 1d). We utilized 12 gRNAs targeting a specific region at 1 bp intervals and 8 gRNAs distributed across the substrate (Sup Fig. 4). Between the two experiments, we observed near-complete cleavage of the substrate for 19 of 20 gRNAs, with the lone gRNA resulting in approximately $50 \%$ substrate digestion (Figs. 1c, 1d). In comparison, wild-type (WT) SpCas 9 digested only 4 of these 20 sites to near-completion (>80\% digestion; Sup Figs. 4c and $4 \mathrm{f}$ ). These results provided evidence that SpRY could act as a potent PAM-agnostic endonuclease in vitro. 
Intrigued by the PAMless nature of SpRYgests, we performed a large comparison of WT SpCas9, SpG (an SpCas9 variant previously engineered to target sites with NGN PAMs ${ }^{5}$ ), and SpRY using 64 additional gRNAs targeting a range of sites bearing all $2^{\text {nd }} / 3^{\text {rd }} / 4^{\text {th }}$ position combinations of an NNNN PAM (Fig. 1e and Sup. Figs. 5a-c). Similar to previous reports ${ }^{6,7}$, WT SpCas9 efficiently digested substrates when programmed with gRNAs targeting sites harboring NGG PAMs, and sometimes exhibited activity against sites with NAG, NGA, or shifted NNGG PAMs (Fig. 1e and Sup. Fig. 5a). SpG recapitulated its preference to edit substrates with NGN PAMs (Fig. 1e and Sup. Fig. 5b). Finally, although an NRN PAM preference was observed with SpRY in mammalian cells $^{5}, S p R Y$ digested the substrate to near-completion when using 59 of 64 gRNAs, partially digested the substrate with 2 gRNAs, and exhibited low-to-no activity with the remaining 3 (Fig. 1e and Sup. Fig. 5c). Together, $93 \%(78 / 84)$ of the gRNAs initially used in these two sets of SpRYgests led to extensive cleavage.
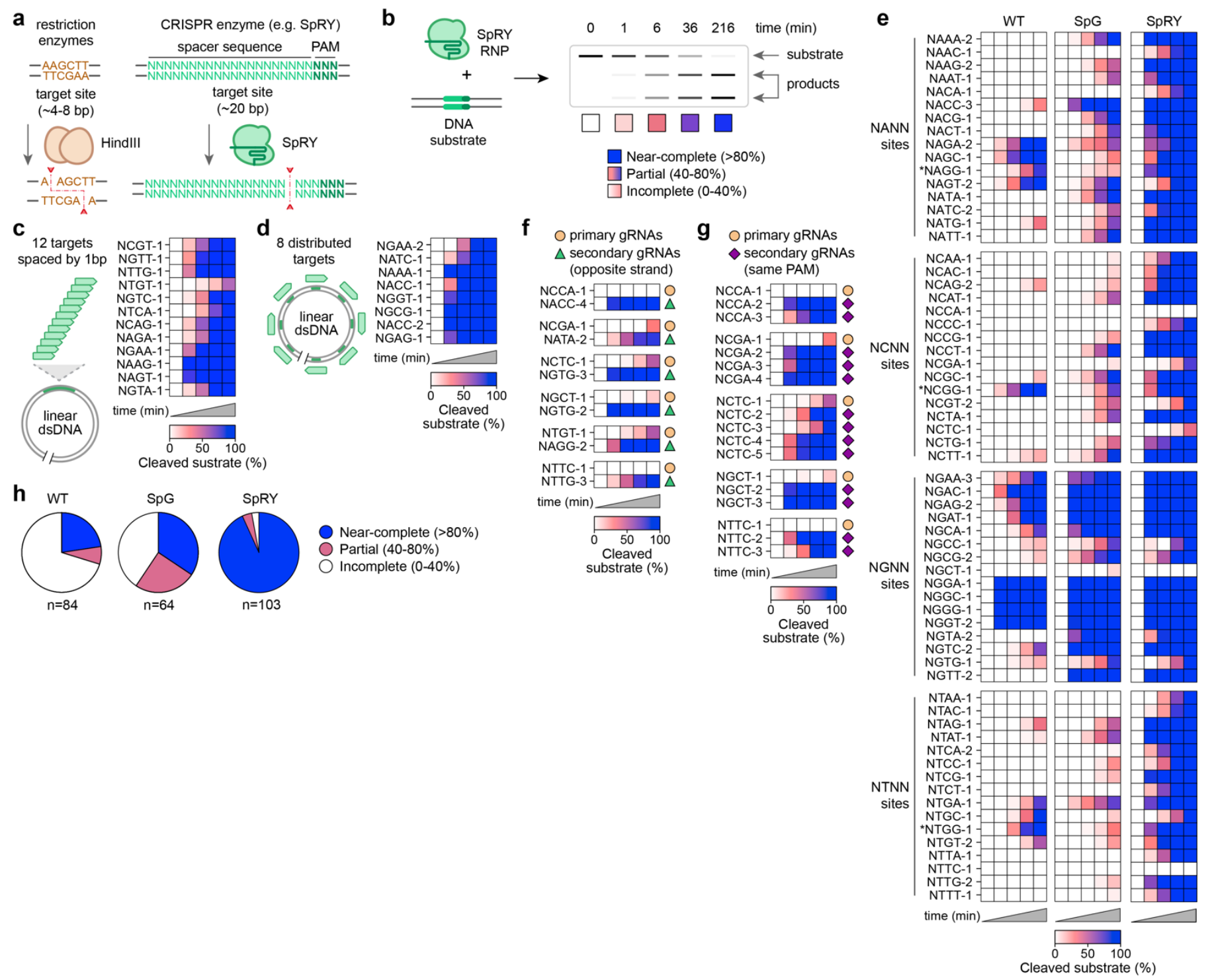

Fig. 1: Characterization of SpRYgest in vitro cleavage efficiencies. (a) Comparison of restriction enzymes (REs) that require fixed 4-8 nt motifs and the near-PAMless Cas9 variant, named SpRY, that can target and 
bioRxiv preprint doi: https://doi.org/10.1101/2022.01.11.474553; this version posted January $12,2022$. The copyright holder for this preprint

(which was not certified by peer review) is the author/funder, who has granted bioRxiv a license to display the preprint in perpetuity. It is made available under aCC-BY-NC-ND 4.0 International license.

cleave DNA substrates without sequence constraints. (b) Illustration of SpRYgest in vitro cleavage reaction workflow. Categorization of substrate cleavage is determined at the final timepoint, judged as near-complete (>80\%), partial (40-80\%), or incomplete (<40\%) digests. (c, d) Initial SpRYgest experiments assessing the DNA cleavage efficiency of $S p R Y$ against a linearized plasmid substrate by targeting a specific region at 1 bp intervals and 8 gRNAs designed to be distributed across the substrate (panels $\boldsymbol{c}$ and $\boldsymbol{d}$, respectively). (e) Comparison of the in vitro cleavage efficiencies of WT, SpG, and SpRY across 64 target sites representing all $2^{\text {nd }} / 3^{\text {rd }} / 4^{\text {th }}$ position combinations of an NNNN PAM. Sites with a shifted NNGG PAM are indicated with an asterisk. (f, $\boldsymbol{g})$ SpRYgest results using additional secondary gRNAs for primary sites in panels 1c and 1e for which partial or incomplete cleavage was observed. Secondary gRNAs were designed to target the opposite DNA strand placing the DSB at the same position as the primary gRNA (panel f), or to target different sites (spacers) but bearing the same PAMs as the primary gRNA (panel g). (h) Summary of the proportion of gRNAs that led to near-complete, partial, or incomplete substrate cleavage when using WT, SpG and SpRY. For panels $\mathbf{c}-\mathbf{g}$, cleavage of DNA substrates was quantified by capillary electrophoresis; mean shown for $n=3$.

Next, we investigated potential causes for incomplete substrate digestion with SpRY. First, for sites where the primary gRNA exhibited partial or incomplete cleavage, we tested the ability of a secondary gRNA targeted to the opposite strand to generate a DSB at the exact same location (Fig. 1f, Sup. Figs. 6a and 6b). This strategy generates the same DSB but via a different target site and gRNA. We assessed the opposite-strand secondary gRNA approach for 6 primary gRNAs that did not reach $>80 \%$ completion. Importantly, we observed $>80 \%$ cleavage for 5 of 6 new secondary gRNAs targeted to the opposite strand and $78 \%$ cleavage for the $6^{\text {th }}$ gRNA (Fig. $1 \mathrm{f}$ and Sup. Fig. 6b), identifying a strategy to overcome low-activity gRNAs. Next, we explored whether low-activity sites could be attributed primarily to a spacer- or PAM-specific source. We tested additional gRNAs targeted to new sites/spacers bearing some of the PAMs that initially resulted in incomplete cleavage (Fig. 1g and Sup. Fig. 6c). For all 13 new gRNAs, we observed near-complete substrate cleavage, suggesting that the PAM is not a primary determinant for incompletely digested sites and that SpRY is generally PAMless in vitro. Collectively, our combined results using 103 gRNAs reveal the flexibility and effectiveness of SpRY for in vitro digests, with $93.2 \%$ of SpRYgests achieving near-complete substrate digestion, an efficiency dramatically higher than for WT SpCas9 or SpG ${ }^{5}$ (Fig. 1h).

The flexibility to generate DSBs at specific positions within plasmid substrates holds promise to simplify, accelerate, and improve the precision of molecular cloning applications. As an alternative to REs, another option aside from SpRYgest is the use of DNA-guided prokaryotic Argonaute (Ago) proteins ${ }^{8,9}$. To compare an Ago protein with SpRY, we initially examined the ability of commercially available Thermus thermophilus Argonaute (TtAgo) to generate custom DNA breaks when programmed with pairs of ssDNA guides (Sup. Figs. 1e and 7a). In experiments adhering to the restrictive target site design considerations for TtAgo (Sup. Fig. 7b and Sup. Note 2) and despite performing metal ion and enzyme dose optimizations (Sup. Figs. 7c and 7d, respectively), 
TtAgo was only able to cleave 2 of 5 substrates to near-completion (Fig. 2a and Sup. Fig. 7e). By comparison, all ten SpRYgests targeting either strand for each of the five TtAgo sites reached near-complete cleavage (Fig. 2a and Sup. Fig. 7e). We then tested TtAgo against two positive control sites and the 20 sites that we initially examined with SpRY (where 19/20 resulted in near-complete digestion; Figs. 1c and 1d). None of these 20 sites accommodated the restrictive TtAgo design requirements and we did not observe evidence of DNA cleavage at any of the 20 sites (Fig. 2b and Sup. Fig. 7f). Given that TtAgo fully digested the DNA substrate for only $8 \%$ of sites examined, at least under our current optimized conditions, TtAgo cannot generate DSBs in vitro as effectively as SpRY (Fig. 2b).
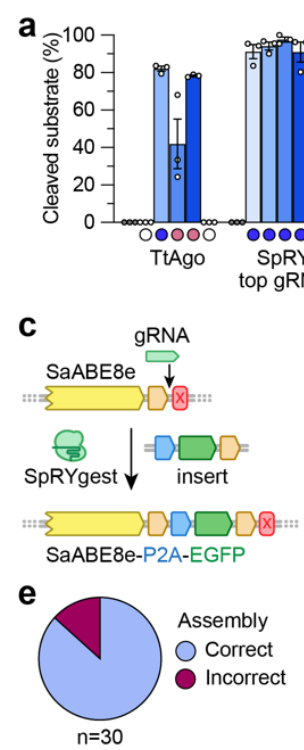

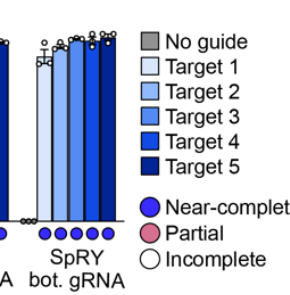

d gRNA-1 gRNA-2

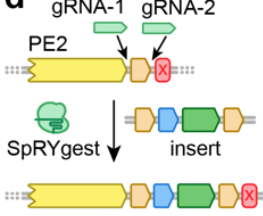

f

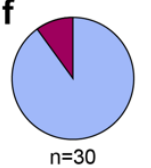

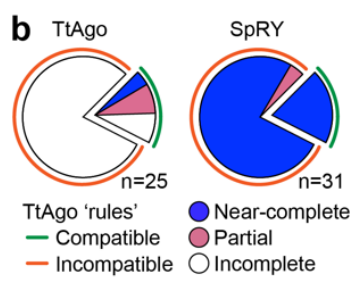

g gRNA
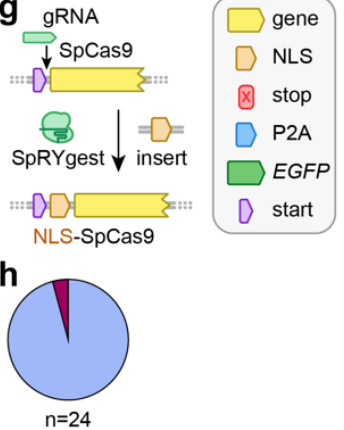

Fig. 2: Molecular cloning via SpRYgest. (a) Comparison of the DNA cleavage efficiencies of TtAgo and SpRY across 5 sites designed to adhere to TtAgo guide requirements. TtAgo reactions were performed with pairs of 5'P-ssDNA guides. TtAgo and SpRYgest reactions were performed for 60 and 216 minutes, respectively. Individual datapoints, mean, and s.e.m. shown for $n=3$. (b) Proportion of TtAgo and SpRY guides that led to nearly complete, partial, or incomplete cleavage on the 20 target sites from Figs. 1c and 1d (see TtAgo results in Supplementary Fig. 7f) and the 5 sites from Fig. 2a. For panels a and b, cleavage of DNA substrates was quantified by capillary electrophoresis; mean shown for $n=3$. (c, d) Schematics of the SpRYgest strategies to add P2A-EGFP sequences to SaCas9-ABE8e and PE2 via single and double SpRYgests, panels $\boldsymbol{c}$ and $\boldsymbol{d}$, respectively. $(\mathbf{e}, \boldsymbol{f})$ Proportion of clones for which correct addition of the P2A-EGFP sequence was confirmed by Sanger sequencing for SaCas9-ABE8e and PE2 strategies, panels e and $\boldsymbol{f}$, respectively. (g) Schematic of the SpRYgest strategy to add an NLS to the N-terminal end of SpCas9. (h) Proportion of clones for which correct addition of the N-terminal NLS was confirmed by Sanger sequencing. 
To evaluate the practical utility of SpRYgests for molecular cloning applications, we first scaled up our cleavage reactions from ng to $\mu \mathrm{g}$ quantities of DNA substrate. Because these conditions necessitated the use large quantities of SpRY, we overexpressed and purified SpRY from E. coli (Sup. Fig. 8). We initially tested purified SpRY in vitro using 3 different gRNAs targeting sites with NAT, NCA, and NGG PAMs across 9 different temperatures. Our data revealed that, consistent with prior reports for WT SpCas 9 , reactions at $37{ }^{\circ} \mathrm{C}$ were optimal for SpRY (Sup. Fig. 9a). The cleavage efficiencies of these 3 gRNAs with purified SpRY were consistent with our previous results using SpRY from a human cell lysate (Sup. Fig. 9b). For sites that exhibited lower activities using the SpRY from lysate, SpRY protein often improved editing efficiency (Sup. Fig. 9c). Upon scaling up the cleavage reactions to $\mu \mathrm{g}$ amounts of DNA substrate, we sometimes observed non-specific degradation of the digestion products (Sup. Fig. 10a). Reactions with gRNA-only conditions led to non-specific nicking of the supercoiled plasmid substrate, suggesting carry-forward of DNase from template removal during the IVT reaction (Sup. Figs. 10a and 10b). Omission of the of the DNase treatment during gRNA synthesis, or use of chemically synthesized gRNAs, eliminated the non-specific degradation of the SpRYgested DNA products (Sup. Fig. 10c).

We then explored the potential of SpRYgests to perform routine cloning applications where unique restriction sites were not available. First, we sought to precisely insert long $\sim 1 \mathrm{~kb}$ P2A-EGFP sequences into plasmids encoding two different genome editors, SaCas9-ABE8 $\mathrm{e}^{10}$ and a prime editor ${ }^{11}$ (PE2) (Figs. 2c and 2d, respectively). To do so, we performed single and double gRNA SpRYgests using $4 \mu \mathrm{g}$ of supercoiled plasmid substrate (Sup. Figs. 11a-11d). For both reactions, complete SpRYgestion was observed. Conversely, no cleavage was observed when using TtAgo targeted to these sites (Sup. Fig. 11e). We then generated a PCR product encoding the P2A-EGFP sequence and cloned it into the digested plasmids via isothermal assembly ${ }^{12}$ (Sup. Figs. 11a and 11b). Of the 30 resulting clones that we sequenced for each assembly, 26 and 27 were correct (Figs. 2e and 2f). In another molecular cloning reaction, we performed a single SpRYgest of the Nterminus of a Cas9 expression plasmid to add a nuclear localization sequence (NLS) (Fig. 2g and Sup. Fig. 12). Isothermal assembly using a short PCR product was extremely efficient, leading to 23 out of 24 clones assembling correctly (Fig. $2 \mathrm{~h}$ ). Together, SpRYgests simplified these three practical and exemplary cloning experiments with assembly efficiencies comparable to typical RE-based digests.

Like genome editing experiments, off-target cleavage of closely related sequences could manifest in SpRYgests. When cloning the SaCas9-ABE8e-P2A-EGFP plasmid, we observed evidence of a very low-level secondary set of products likely caused by an off-target DSB (Sup. Fig. 11c). Closer inspection of the target site revealed that the off-target cleavage was the result of utilizing a gRNA that overlapped an NLS, for which there was a second NLS with high sequence homology elsewhere in the plasmid, bearing 3 mismatches (Sup. Figs. 13a and 13b). We were able to completely mitigate off-target cleavage by utilizing a secondary gRNA targeted to the more unique sequence on the opposite strand (Sup. Figs. 13c and 13d). Another potential method to eliminate offtarget editing is to utilize a high-fidelity version of SpRY, SpRY-HF1 ${ }^{5,13}$ (Sup. Fig. 13e), that has previously been 
shown to be more sensitive to mismatches. While SpRY-HF1 did not completely prevent off-target editing when utilizing the NLS-targeted gRNA at the final digestion timepoint (Sup. Fig. 13f), a comparison of SpRY and SpRY-HF1 when programmed with mismatched gRNAs revealed that in certain cases, SpRY-HF1 can reduce off-target cleavage in vitro, especially at earlier reaction timepoints (Sup. Fig. 13g).

Another potential application of performing a SpRYgest is to generate plasmid libraries bearing customized regions at any location rapidly and cost-effectively. As a proof-of-concept, we sought to investigate biological properties of SpCas 9 by generating two saturation mutagenesis libraries with randomized nucleotides in regions of SpCas9 that are critical for either the catalytic activity ${ }^{14}$ (Fig. 3a and Sup. Fig. 14a) or the PAM preference ${ }^{7,15}$ of SpCas9 (Fig. 3b and Sup. Fig. 14b). We linearized an SpCas9-encoding plasmid via a double-SpRYgest and performed isothermal assembly reactions with ssDNA oligonucleotides encoding degenerate NNS codons (where ' $N$ ' is any nucleotide and ' $S$ ' is $G$ or $C$ ). Via both Sanger and next-generation sequencing, we observed balanced representation of nucleotides in the modified positions (Figs. $3 \mathrm{c}$ and $\mathbf{3 d}$ ). The libraries also contained intentionally coded silent substitutions to enable assessment of library construction efficiency, which were introduced at $>99.8 \%$ suggesting highly effective synthesis with minimal background (Figs. $3 c$ and $3 d$ ).

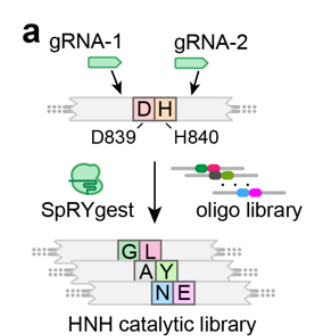

f
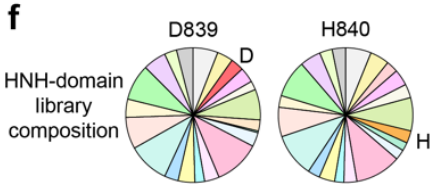

$\mathrm{n}=12,378$ (NGS)

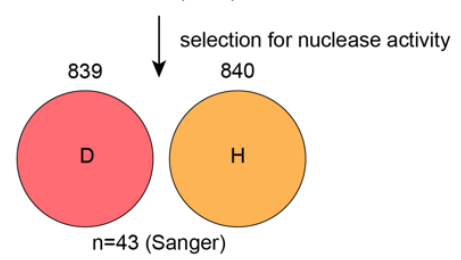

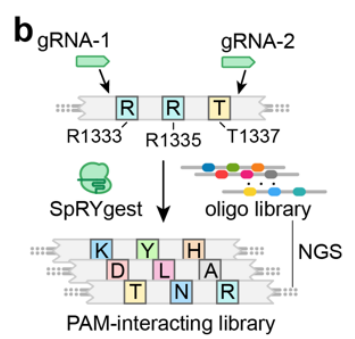
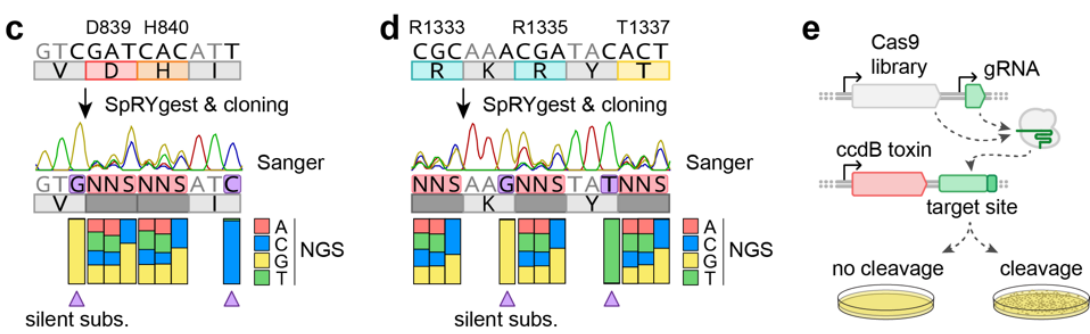

g

$\square \mathrm{A} \square \mathrm{I} \square \mathrm{R}$
$\square \mathrm{C} \square \mathrm{K} \square \mathrm{S}$ $\square \mathrm{D} \square \mathrm{L} \quad \mathrm{T}$ $\square \mathrm{E} \square \mathrm{M} \square \mathrm{V}$ $\square \mathrm{F} \square \mathrm{N} \square$ $\square \mathrm{G} \square \mathrm{P} \square \mathrm{Y}$ $\square \mathrm{H} \square \mathrm{Q} \square \mathrm{X}$
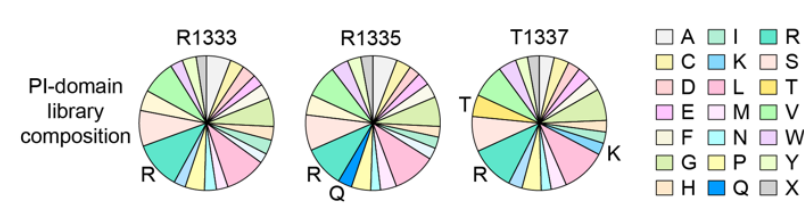

$\mathrm{n}=409,221$ (NGS)
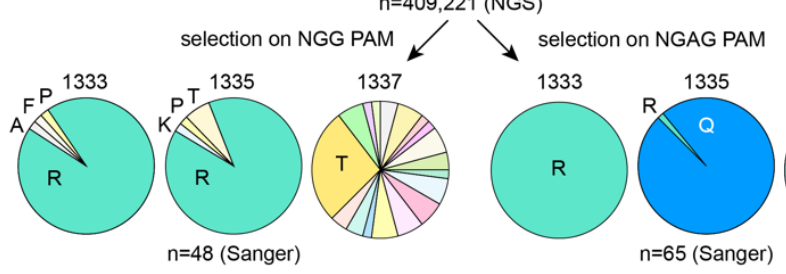

Fig. 3: Generation of saturation mutagenesis libraries via SpRYgest. (a,b) Schematics of SpRYgest strategies to generate saturation mutagenesis libraries of SpCas9 residues important for catalytic activity (panel a) and NGG PAM preference (panel b). (c,d) Sanger sequencing traces and next-generation sequencing results from the libraries, illustrating the nucleotide diversity at mutated residues for the HNH-catalytic and PAMinteracting $(P I)$ domain libraries, panels $\boldsymbol{c}$ and $\boldsymbol{d}$, respectively. Recoded silent substitutions were intentionally included in the library to assess construction efficiency (highlighted in purple and indicated with a triangle). (e) Schematic of the bacterial positive selection assay ${ }^{7,16,17}$, which permits selection of cleavage competent SpCas9 
bioRxiv preprint doi: https://doi.org/10.1101/2022.01.11.474553; this version posted January 12, 2022. The copyright holder for this preprint

(which was not certified by peer review) is the author/funder, who has granted bioRxiv a license to display the preprint in perpetuity. It is made available under aCC-BY-NC-ND 4.0 International license.

enzymes from saturation mutagenesis libraries. Colonies survive on selective media only when SpCas9 and a gRNA cleave a target site on the toxic plasmid. Mutated regions of SpCas 9 can be sequenced from the plasmids harbored within surviving colonies. $(\boldsymbol{f}, \boldsymbol{g})$ Post-selection results for cleavage competent SpCas 9 variants from the catalytic domain $\mathrm{HNH}$ residue library (panel f), or from the PI domain library selected against toxic plasmids harboring target sites with NGG and NGAG PAMs (left and right sides of panels $\mathbf{g}$, respectively). Pie charts illustrate the distribution of amino acids at each position in the pre-selection libraries (via NGS) and post-selection libraries (via Sanger sequencing of individual clones) in the top and bottom panels, respectively.

We then subjected these SpRYgest-constructed libraries to a previously described bacterial positiveselection $^{7,16,17}$. Survival of colonies harboring transformed plasmids is dependent on the ability of SpCas 9 to cleave a target site encoded within a selection plasmid that expresses a toxic gene (Fig. 3e). First, we performed experiments using the catalytic domain library that was varied at conserved HNH nuclease positions D839 and H840 (Figs. 3a and 3c). Selection for cleavage-competent clones revealed that, as expected ${ }^{18}$, only SpCas 9 variants encoding D839 and H840 were cleavage competent (Fig. 3f). We also evaluated the SpCas9 PAMinteracting (PI) domain library that was varied at amino acids critical for PAM recognition including R1333, R1335, and T1337 (Fig. 3b and 3d). Selection of the library against a toxic plasmid encoding a target site with an NGG PAM led mostly to clones with R1333 and R1335, the two amino acid sidechains known to be important for specifying the guanines of the PAM $^{15}$ (Fig. $3 g$ ). Interestingly, a variety of amino acids were observed at position 1337, though the native T1337 was enriched relative to the others. An additional selection with the same library against a target encoding an NGAG PAM led to enrichment of variants with R1333, R1335Q, and T1337R/K (Fig. 3g), consistent with expectations for amino acids that facilitate recognition of this non-canonical PAM $^{19,20}$ (and previous results with the engineered variants SpCas9-VQR and SpCas9-VRQR ${ }^{7,13}$ ).

Finally, for SpRYgests to be more readily implemented, we sought to simplify and expedite the gRNA synthesis protocol (Sup. Fig. 15a). To do so, we experimented with various one-pot gRNA synthesis conditions and methods that require minimal hands-on time by combining the template generation and IVT steps (Sup. Figs. 15a-15d and Sup. Note 3). We identified conditions with shortened IVT reaction times (<4 hours) that generate high gRNA yields (Sup. Figs. 15b and 15c). These optimized one-pot reaction conditions reduce SpRYgest hands-on times by approximately 3.5-fold, leading to workflows that are more similar to RE digests (Fig. 4a). Importantly, one-pot gRNA IVT reactions performed at two different scales for four different gRNAs all supported complete digestion of a plasmid substrate (Fig. 4b), reducing the enzymatic cost of gRNA synthesis by $300 \%$ to approximately $\$ 2.33$ per gRNA (Sup. Note 3). The optimization of one-pot gRNA synthesis methods dramatically minimizes hands-on time, minimizes cost by scaling-down gRNA reactions, and makes the SpRYgest workflow more similar to other molecular cloning experiments (Fig. 4a and Sup. Fig. 16). 
bioRxiv preprint doi: https://doi.org/10.1101/2022.01.11.474553; this version posted January 12, 2022. The copyright holder for this preprint (which was not certified by peer review) is the author/funder, who has granted bioRxiv a license to display the preprint in perpetuity. It is made available under aCC-BY-NC-ND 4.0 International license.
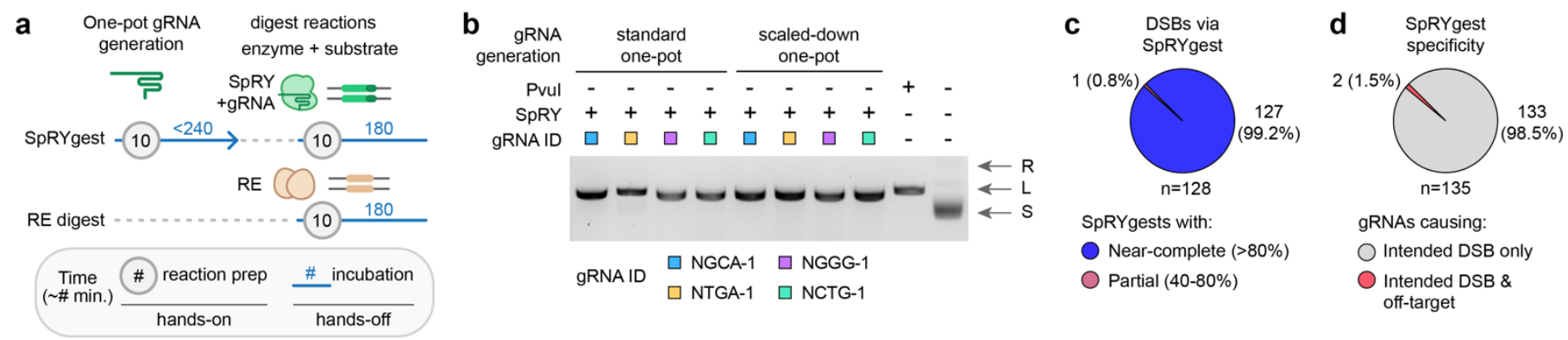

Fig. 4: Optimization of rapid, efficient, and specific SpRYgest reactions. (a) Comparison of the hands-on and hands-off times of optimized SpRYgests (with gRNAs generated via one-pot reactions) versus RE digests. Approximate times in minutes are shown; lines not drawn to scale. The incubation time for one-pot gRNA generation can be shortened as needed (see Sup. Fig. 15b). (b) Agarose gel of SpRYgest reactions performed with gRNAs taken directly from standard or scaled-down one-pot gRNA generation reactions (20 $\mu \mathrm{L}$ or $5 \mu \mathrm{L}$ onepot reactions, respectively). Plasmid conformations are: $R$, relaxed; L, linear; S, supercoiled. (c) Proportion of unique DSBs that were successfully generated via SpRYgest in all experiments of this study. (d) Proportion of gRNAs for which off-target cleavage products were detected during SpRYgests with gRNAs used in all experiments of this study. 


\section{Discussion:}

Here we establish a new paradigm of unconstrained DNA manipulation in vitro using a CRISPR-Cas enzyme, SpRY. We discover that SpRY is essentially PAMless when deployed in molecular cleavage reactions, and leverage this property for highly precise DNA digestion. With SpRYgests, standard and more complex cloning reactions are simplified, including the generation of saturation mutagenesis libraries to interrogate biological properties of impactful proteins (as demonstrated by our analysis of two SpCas9 domains). The PAMless nature of SpRY suggests that the NRN>NYN PAM preference we previously observed in human cells ${ }^{5}$ is less prominent under defined in vitro conditions. This is likely due to the fact that the 'genome' size of a plasmid is dramatically different than that of a eukaryotic organism, which influences SpRY target search time and how the preferred PAMs of a Cas variant are encountered and engaged ${ }^{21-23}$.

Across all experiments, we were able to generate a DNA break at every position that we sought to (Fig. 4c). For cases where the primary gRNA failed or was inefficient, reactions with the secondary gRNA that positions the DSB at the same position cleaved the substrate to near-completion (>80\%) in all but one case ( where this single gRNA reached $\sim 78 \%$ substrate digestion; Fig. 4c). We anticipate that the digestion efficiency can be further improved and expedited by simply increasing the amount of SpRY-gRNA complex included in the SpRYgest reaction, including for weaker gRNAs that don't initially lead to complete cleavage. Our results also indicate that the substrate preferences of Ago proteins ${ }^{8,9}$ limit their applicability for DNA digests.

The unconstrained targeting range of SpRY eliminates the need for a large repertoire of REs. Even with a comprehensive catalog of REs, rarely does a single cutter RE site exist at the intended location of the DNA modification. SpRYgests require only a single source of the SpRY protein (versus dozens of REs) and a gRNA, which can be generated by IVT (Fig. 4a and Sup. Fig. 15). To streamline the IVT process, we optimized a rapid, simple, and affordable one-pot gRNA generation method, the product of which can be added directly to a SpRYgest reaction (Fig. 4b and Sup. Note 3). Despite the already minimal cost and rapid workflow of SpRYgest reactions, further optimization is possible (e.g. of the IVT reaction ${ }^{24}$ ). SpRYgest timelines are not substantially different than traditional cloning workflows, which are similarly dependent on the design and receipt of custom oligonucleotides (Sup. Fig. 16). We provide additional guidance on performing SpRYgests and considerations for experimental design (e.g. omitting the DNase treatment during IVT of gRNAs; see Sup. Note 4). Since Cas9 enzymes predominantly leave blunt DNA breaks, SpRYgests do not leave overhangs typical of most REs (a property largely obviated by the use of isothermal assembly ${ }^{12}$ ). However, paired SpRY nickases could in principle be utilized to generate cohesive 'sticky' ends as needed.

Over the course of this study when performing 136 separate SpRYgests, we observed only very low level offtarget cleavage for 2 gRNAs (both of which were attributable to related sequences in the plasmids; Fig. 4d and 
Sup Figs. 13b and 13h). These results indicate that achieving a single intended digestion product via SpRYgest is possible when considering other closely related sequences in the plasmid, and that simple methods can be used to mitigate off-target cleavage (by identifying when targeting the opposite strand would have fewer predicted off-target sites or by using SpRY-HF1, both of which can reduce or eliminate off-target cleavage). To prospectively identify gRNAs with closely matched off-target sites in DNA substrates, we have developed a webbased tool called SpOT-check (SpRYgest Off-Target checker; see Sup. Note 5).

Beyond a general usefulness for standard cloning, SpRYgests can improve various applications including saturation mutagenesis, domain minimization or shuffling ${ }^{25}$, depleting unwanted sequences from sequencing libraries ${ }^{26}$, target enrichment in sequencing protocols ${ }^{27}$, to generate more precisely terminated IVT templates, and other uses for the detection or diagnosis of infectious and genetic diseases. The flexibility of SpRYgests to generate DSBs at specific positions within DNA substrates holds promise to simplify, accelerate, and improve the precision of molecular applications, many of which not previously possible when using REs. 


\section{Acknowledgements:}

We thank R.T. Walton for helpful suggestions and S. Mahendraker for assistance developing the web version of SpOT-check. K.A.C. is supported by a Massachusetts General Hospital (MGH) Fund for Medical Discovery (FMD) Fundamental Research Fellowship Award. B.P.K. acknowledges support from an MGH Executive Committee on Research Howard M. Goodman Fellowship.

\section{Author Contributions:}

K.A.C. and B.P.K. conceived of and designed the study. K.A.C., J.A.G., R.A.S., R.M.D., H.E.S. and L.M. performed experiments. M.M. and G.B.R. expressed and purified SpRY and SpRY-HF1. R.A.S. designed and wrote SpOT-check. All authors analyzed data. K.A.C. and B.P.K. wrote the manuscript draft and finalized the manuscript with input from all authors.

\section{Corresponding Author:}

Correspondence should be addressed to B.P. Kleinstiver (bkleinstiver@mgh.harvard.edu)

\section{Competing Interests Statement:}

K.A.C. and B.P.K are inventors on patents and/or patent applications filed by Mass General Brigham that describe genome engineering technologies. B.P.K. is a consultant for Avectas Inc., EcoR1 capital, and ElevateBio, and is an advisor to Acrigen Biosciences and Life Edit Therapeutics.

\section{Data Availability:}

All primary data will be made available as a Supplementary Table. 


\section{Methods:}

\section{Plasmids and oligonucleotides}

Descriptions and Addgene IDs for all plasmids used in this study are available in Sup. Table 1; new plasmids have been deposited with Addgene (https://www.addgene.org/Benjamin Kleinstiver/). A list of all SpRYgest target sites is provided in Sup. Table 2 that includes spacer sequences, PAMs, and gRNA generation methods. Oligonucleotide sequences and descriptions are available in Sup. Table 3. Target sites for TtAgo are listed in Sup. Table 4. Additional details for plasmids and oligonucleotides (oligos) are provided below in the respective sections. The SpOT-check computed off-target profiles for all gRNAs used in this study are available in Sup. Table 5.

\section{Human cell culture}

Human HEK 293T cells (ATCC) were cultured in Dulbecco's Modified Eagle Medium (DMEM) supplemented with $10 \%$ heat-inactivated FBS (HI-FBS) and 1\% penicillin/streptomycin. The supernatant media from cell cultures was analyzed monthly for the presence of mycoplasma using MycoAlert PLUS (Lonza).

\section{Expression of and normalization of SpCas9-containing human cell lysates}

Expression plasmids encoding WT SpCas9, SpG, and SpRY each with a -P2A-EGFP signal (RTW3027, RTW4177 and RTW4830, respectively) were used to generate human cell lysates containing SpCas9 proteins. Approximately 20-24 hours prior to transfection, $1.5 \times 10^{5}$ HEK $293 \mathrm{~T}$ cells were seeded in 24-well plates. Transfections containing $500 \mathrm{ng}$ of human codon optimized nuclease expression plasmid and $1.5 \mu \mathrm{L}$ TransIT$\mathrm{X} 2$ were mixed in a total volume of $50 \mu \mathrm{L}$ of Opti-MEM, incubated at room temperature for 15 minutes, and added to the cells. The lysate was harvested after 48 hours by discarding the media and resuspending the cells in 100 $\mu \mathrm{L}$ of gentle lysis buffer (containing 1X SIGMAFAST Protease Inhibitor Cocktail, EDTA-Free (Sigma), $20 \mathrm{mM}$ Hepes $\mathrm{pH} 7.5,100 \mathrm{mM} \mathrm{KCl}, 5 \mathrm{mM} \mathrm{MgCl}_{2}, 5 \%$ glycerol, $1 \mathrm{mM}$ DTT, and $0.1 \%$ Triton X-100). The amount of SpCas 9 protein was approximated from the whole-cell lysate based on EGFP fluorescence. SpCas 9 lysates were normalized to $180 \mathrm{nM}$ fluorescien (Sigma) based on a standard curve. Fluorescence was measured in 384well plates on a DTX 880 Multimode Plate Reader (Beckman Coulter) with $\lambda_{\mathrm{ex}}=485 \mathrm{~nm}$ and $\lambda_{\mathrm{em}}=535 \mathrm{~nm}$.

\section{Production of gRNAs}

The DNA substrates required to transcribe gRNAs were generated via two methods. First, plasmids for IVT of SpCas9 gRNAs were generated by annealing and ligating duplexed oligos (see Sup. Table 3) corresponding to spacer sequences into Bsal-digested MSP3485 for T7 promoter-driven transcription of gRNAs. The derivative pT7-spacer-gRNA plasmids were digested with HindIII (NEB) to permit run-off transcription near the 3' end of the SpCas9 gRNA. Secondly, oligo-derived DNA templates for IVT were generated by combining a target specific oligo (encoding a T7 promoter, spacer sequence, and partial sequence of the SpCas 9 crRNA) and a common SpCas9 gRNA scaffold oligo (oKAC682), and then incubating with either Klenow Fragment ( $3^{\prime} \rightarrow 5^{\prime}$ exo-) (New 
England Biolabs (NEB), M0212L) in $1 \times$ NEBuffer 2 at $37^{\circ} \mathrm{C}$ for 30 minutes, or $Q 5$ polymerase (NEB) using the following program: 2 minutes $98^{\circ} \mathrm{C} ; 5$ cycles of $\left(10\right.$ seconds $98^{\circ} \mathrm{C}, 10$ seconds $65^{\circ} \mathrm{C}, 30$ seconds $\left.72{ }^{\circ} \mathrm{C}\right) ; 5$ minutes $72{ }^{\circ} \mathrm{C}$. Plasmid or oligo-derived transcription templates were cleaned up using a MinElute PCR Purification Kit (Qiagen). SpCas 9 gRNAs were transcribed at $37^{\circ} \mathrm{C}$ for 16 hours using the T7 RiboMAX Express Large Scale RNA Production Kit (Promega). For gRNAs utilized in in vitro cleavage reactions containing SpRY from human cell lysates, the $37^{\circ} \mathrm{C}$ incubation was followed by the addition of $1 \mu \mathrm{L} R Q 1 \mathrm{DNase}$ at $37^{\circ} \mathrm{C}$ for 15 minutes to degrade the DNA template. The DNase treatment step was omitted when preparing most gRNAs utilized for scaled-up SpRYgest reactions with purified SpRY protein. Following transcription and optional DNase treatment, gRNAs were purified using paramagnetic beads (prepared as previously described ${ }^{28}$; GE Healthcare Sera-Mag SpeedBeads from Fisher Scientific, washed in 0.1X TE and suspended in 20\% PEG-8000 (w/v), $1.5 \mathrm{M}$ $\mathrm{NaCl}, 10 \mathrm{mM}$ Tris- $\mathrm{HCl} \mathrm{pH} 8,1 \mathrm{mM}$ EDTA pH 8 and $0.05 \%$ Tween20) and refolded by heating to $90{ }^{\circ} \mathrm{C}$ for 5 minutes and then cooling to room temperature at $1{ }^{\circ} \mathrm{C}$ every 2 seconds. Synthetic gRNAs were purchased from Synthego.

For one-pot gRNA IVT reactions, we utilized two general methods. First, gRNAs were generated using the EnGen sgRNA Synthesis Kit (NEB, E3322S) according to the manufacturer recommended protocol, or the EnGen sgRNA Synthesis Kit with increased oligo concentrations (final concentrations of $0.75 \mu \mathrm{M}$ target-specific oligo and $0.75 \mu \mathrm{M}$ common SpCas9 gRNA scaffold oligo (oKAC682)). The DNase step was omitted. Second, we also optimized a separate one-pot gRNA synthesis method using other commercial reagents. In this second method, $20 \mu \mathrm{L}$ one-pot reactions were assembled containing final amounts or concentrations of $2.5 \mathrm{U}$ Klenow Fragment ( $3^{\prime} \rightarrow 5^{\prime}$ exo-), target-specific oligo at 0.5 or $1.5 \mu \mathrm{M}$ (for standard or scaled-up reactions, respectively), common SpCas9 gRNA scaffold oligonucleotide (oKAC682) at 0.25 or $0.75 \mu \mathrm{M}$ (for standard or scaled-up reactions, respectively), $125 \mu \mathrm{M}$ dNTPs, 1x RiboMAX Express T7 Buffer (Promega, P1320), and $2 \mu \mathrm{L}$ T7 Express Enzyme Mix (Promega, P1320) and incubated at $37^{\circ} \mathrm{C}$ for 4 hours unless otherwise indicated. Appropriately scaled $5 \mu \mathrm{L}$ reactions were assembled for smaller-scale one-pot reactions. For one-pot gRNAs used in SpRYgest reactions, the Promega recommended RQ1 DNase treatment was omitted and no clean-up of the gRNA was performed. To quantify gRNA yield, separate IVT reactions were performed that included the RQ1 DNase step and were purified using paramagnetic beads. Note that gRNA yield will vary based on incubation time.

\section{Expression and purification of SpRY and SpRY-HF1 proteins}

E. coli codon optimized SpRY and SpRY-HF1 coding sequences including an N-terminal MKIEE tag and Cterminal SV40 NLS and 6x histidine tag were synthesized (GenScript, NJ, USA) and cloned into pET28 expression vectors. The SpRY and SpRY-HF1 expression constructs and were used to express and purify the proteins as described previously ${ }^{29}$. Briefly, E. coli strain NiCo21(DE3) (C2529H from NEB) harbouring the recombinant construct was grown in 1-2 L of LB medium with $40 \mu \mathrm{g} / \mathrm{mL}$ Kanamycin at $30^{\circ} \mathrm{C}$ until mid-log phase. Overexpression of the target protein was induced by adding IPTG to a final concentration of $0.4 \mathrm{mM}$ with shaking 
overnight at $18^{\circ} \mathrm{C}$. Cells were harvested and target protein expression was assessed by SDS PAGE prior to purification. Cells were disrupted by sonication in breakage buffer $(50 \mathrm{mM}$ Tris- $\mathrm{HCl}(\mathrm{pH} 8.0), 300 \mathrm{mM} \mathrm{NaCl}, 1 \mathrm{mM}$ EDTA, 1mM DTT, 2\% (v/v) glycerol) supplemented with PMSF. The supernatant was passed through HiTrap DEAE Sepharose (Cytiva, MA, USA) in column buffer (20mM Tris $(\mathrm{pH} 7.5)$ and 250mM NaCl) followed by subsequent purification on a HisTrap HP column (Cytiva). After 16x column volume wash in buffer containing $20 \mathrm{mM}$ Tris $\mathrm{pH} 7.5,250 \mathrm{mM} \mathrm{NaCl}, 40 \mathrm{mM}$ imidazole, target proteins were eluted using a $40 \mathrm{mM}$ to $750 \mathrm{mM}$ imidazole gradient in the same buffer. Pooled fractions containing the proteins were further purified by loading onto HiTrap heparin HP columns (Cytiva), washed with 6 column volumes of a buffer containing 20mM Tris (pH8.0), 1mM EDTA, and $1 \mathrm{mM}$ DTT, and eluted using a 0.25 to $2 \mathrm{M} \mathrm{NaCl}$ gradient in the same buffer. Pooled fractions were dialyzed in SEC column buffer (20mM HEPES (pH8.0), 250mM KCl, and $1 \mathrm{mM} \mathrm{DTT}$ ) and concentrated using an Amicon® Ultra-15 Centrifugal Filter Unit with $100 \mathrm{kDa}$ molecular weight cut-off . Concentrated fractions were loaded on to a HiLoad 16/600 Superdex 200 pg column (Cytiva) using a $1 \mathrm{~mL}$ sample loop. Size exclusion chromatography was performed in SEC column buffer with a flow rate of $0.5 \mathrm{~mL} / \mathrm{min}$. Eluted fractions were assessed by SDS-PAGE, pooled, dialyzed in storage buffer (20mM Tris (pH7.5), 300mM NaCl, 0.1mM EDTA, $1 \mathrm{mM} \mathrm{DTT}$ and $50 \%(\mathrm{v} / \mathrm{v})$ glycerol), and stored at $-20^{\circ} \mathrm{C}$. Protein concentration was determined by Bradford assay using BSA for standards.

\section{In vitro cleavage reactions using SpCas9 from lysates}

Plasmid KAC833 linearized with HindIII (NEB) was used as the DNA substrate for most in vitro cleavage reactions unless otherwise stated. SpCas9 ribonucleoprotein (RNP) complexes were formed by mixing $9 \mu \mathrm{L}$ of SpCas9-containing normalized whole-cell lysate (normalized to $180 \mathrm{nM}$ Fluorescein) with $11.25 \mathrm{pmol}$ of transcribed or synthetic gRNA, and incubating for 5 minutes at $37^{\circ} \mathrm{C}$. Cleavage reactions were initiated by the addition of $34.82 \mathrm{fmol}$ of linearized plasmid (digested with HindIII (NEB)) and buffer to bring the total reaction volume to $22.5 \mu \mathrm{L}$ with a final composition of $10 \mathrm{mM} \mathrm{HEPES} \mathrm{pH} \mathrm{7.5,150} \mathrm{mM} \mathrm{NaCl}$, and $5 \mathrm{mM} \mathrm{MgCl}$. Reactions were performed at $37^{\circ} \mathrm{C}$ and aliquots were terminated at timepoints of $1,6,36$ and 216 minutes by removing 5 $\mu \mathrm{L}$ aliquots, mixing with $5 \mu \mathrm{L}$ of stop buffer (50 mM EDTA and $2 \mathrm{mg} / \mathrm{ml}$ Proteinase $\mathrm{K}(\mathrm{NEB})$ ), and incubating at room temperature for 10-minutes. Cleavage fragments were purified using paramagnetic beads and quantified via QIAxcel capillary electrophoresis (Qiagen). The relative abundances of substrate and products were analyzed using QIAxcel ScreenGel Software (v1.5.0.16, Qiagen) and plotted using GraphPad Prism 9 (v9.2.0).

\section{In vitro cleavage reactions using purified SpRY}

Small-scale in vitro cleavage reactions were performed as described above, except using 0.6-1 $\mu \mathrm{M}$ purified SpRY per reaction pool instead of $9 \mu \mathrm{L}$ of SpCas9-containing normalized whole-cell lysate $(0.6 \mu \mathrm{M}$ in Sup. Figs. 9a and $9 \mathrm{~b}$ and $1 \mu \mathrm{M}$ in 9c). For scaled-up digests, $4 \mu \mathrm{g}$ of supercoiled plasmid DNA was incubated at $37^{\circ} \mathrm{C}$ for 3 hours with purified SpRY protein at a final concentration of $1 \mu \mathrm{M}$ and IVT gRNA (prepared without DNase treatment) at a final concentration of $2 \mu \mathrm{M}$ in Buffer 3.1 (NEB). Reactions were stopped by the addition of $1 \mu \mathrm{L}$ 
of Proteinase K (NEB) and incubated at room temperature for 15 minutes. Cleavage fragments were resolved by $0.8 \%$ agarose gel electrophoresis with $1 \mu \mathrm{L}$ of $1 \mathrm{~kb}$ Plus DNA Ladder (NEB) and visualized by ethidium bromide staining. Digestion products were purified using a QIAquick Gel Extraction Kit (Qiagen).

\section{Molecular cloning reactions using purified SpRY}

The C-terminal P2A-EGFP sequence was added to SaABE8e or pCMV-PE2 (Addgene IDs 138500 and 132775 , respectively), and the N-terminal BPNLS was added to an SpCas9 plasmid similar to pCMV-T7-SpCas9 (Addgene plasmid ID 139987) via isothermal assembly. Reactions contained approximately $5 \mu \mathrm{L}$ of isothermal assembly mix (prepared similar to as previously described ${ }^{12}$ ) or NEBuilder HiFi (NEB), 0.01 pmol of plasmid linearized via SpRYgest, and $0.03 \mathrm{pmol}$ of PCR product insert in a final volume of $10 \mu \mathrm{L}$, and incubated at $50^{\circ} \mathrm{C}$ for 60 minutes. Cloning reactions were transformed into chemically competent XL1-Blue E. coli cells and grown at $37{ }^{\circ} \mathrm{C}$ for approximately 16 hours. Individual colonies were grown overnight at $37^{\circ} \mathrm{C}$, miniprepped (Qiagen), and fidelity of cloning was verified via Sanger sequencing. Saturation mutagenesis plasmid libraries for were constructed by incubating 0.02 pmol of BPK848 (linearized via SpRYgest) with 0.1 pmol of $\sim 60$ bp ssDNA oligo (either oBK9102 or oBK9104) with $10 \mu \mathrm{L}$ NEBuilder HiFi DNA Assembly Master Mix (NEB) in a final volume of $20 \mu \mathrm{L}$, and incubated at $50{ }^{\circ} \mathrm{C}$ for 15 minutes. NEBuilder reactions were cleaned up via MinElute (Qiagen) and eluted in $10 \mu \mathrm{L}$ water, transformed into $100 \mu \mathrm{L}$ of electrocompetent $\mathrm{XL} 1$-Blue $E$. coli, and recovered in $3 \mathrm{~mL}$ of SOC for $1 \mathrm{~h}$ at $37^{\circ} \mathrm{C}$. Next, $2 \mu \mathrm{L}$ of the transformation recovery media was plated on LB + chloramphenicol, where the number of colonies following overnight at $37^{\circ} \mathrm{C}$ were used to estimate library complexity. The remaining recovery was grown overnight in $150 \mathrm{~mL}$ LB + chloramphenicol and plasmid DNA was isolated by MaxiPrep (Qiagen). The complexity of the SpCas9 catalytic and PAM domain libraries were estimated to be 77,400 and 292,600 respectively. Plasmid libraries were sequenced via Sanger sequencing and NGS. For NGS, PCR amplicons were generated from the plasmids using primer pairs oKAC1589/oKAC1590 (for the catalytic domain) or oKAC1591/oKAC1592 (for the PI domain) and sequenced on a MiSeq (Illumina) to a depth of 12,378 and 409,221 reads for the catalytic and PI domain libraries, respectively. The resulting data was analyzed using CRISPResso $2^{30}$ to generate allele tables.

\section{In vitro cleavage reactions using TtAgo}

For TtAgo reactions, 5'-phosphorylated DNA guides were either purchased from Integrated DNA technologies or generated by incubating an unmodified oligonucleotide with T4 Polynucleotide Kinase (NEB) at $37^{\circ} \mathrm{C}$ for 30 minutes, followed by heat-activation at $65^{\circ} \mathrm{C}$ for $20 \mathrm{~min}$. Complexes of TtAgo programmed with ssDNA guides were prepared by combining final concentrations of 1 pmol TtAgo (NEB) and 2 pmol 5'-phosphorylated ssDNA guides and incubating at $70{ }^{\circ} \mathrm{C}$ for 20 minutes. Cleavage reactions were performed by combining TtAgo complexes with either $79.85 \mathrm{fmol}$ of linearized KAC833 plasmid substrate (digested with Pvul, NEB) or 79.85 fmol supercoiled plasmid DNA (KAC1151 or MNW95) in ThermoPol buffer (NEB) with a final concentration of $10 \mathrm{mM} \mathrm{MgSO}_{4}$. Reactions were performed at $80{ }^{\circ} \mathrm{C}$ for 60 minutes and terminated by the addition of $1 \mu \mathrm{L}$ of 
Proteinase K (NEB). Cleavage fragments from pre-linearized substrates were purified using paramagnetic beads and quantified and analyzed as described above. Cleavage fragments from scaled-up plasmid DNA digests were resolved by $0.8 \%$ agarose gel electrophoresis and visualized by ethidium bromide staining.

\section{Bacterial-based positive selection assay}

Target plasmids for the selection assays were generated by cloning duplexed oligonucleotides into Xbal and Sphl-digested p11-lacY-wtx1 (Addgene ID 69056) ${ }^{16}$ as previously described ${ }^{7}$, which contains an arabinoseinducible ccdB toxin gene. The derivative toxin-expressing plasmids contain target sites harboring either NGG or NGA PAMs (BPK740 and BPK754, respectively). To perform the selections, electrocompetent E. coli BW25141 $(\lambda D E 3)^{17}$ containing a toxin-expressing plasmid were transformed with BPK848-derived plasmids that express the SpCas9 variant libraries (encoding randomized codons in specified positions) in addition to a gRNA, both from separate T7 promoters. Following a 60-minute recovery in SOC media, transformations were spread on LB plates containing either chloramphenicol and $10 \mathrm{mM}$ dextrose (non-selective) or chloramphenicol +10 $\mathrm{mM}$ arabinose (selective). Transformation efficiency was assessed based on colony count from non-selective plates. The catalytic library selection resulted in approximately $9 \mathrm{e} 4$ colonies (from sampling approximately $87 x$ library coverage). The PI domain library selections for NGG PAMs and NGA PAMs resulted in approximately $6 e 5$ and $6.4 e 4$ colonies (from sampling approximately $18 x$ and $2 x$ coverage of the libraries, respectively). Surviving colonies from selective plates were picked as single colonies for miniprep (Qiagen) followed by Sanger sequencing to verify the identities of the mutated amino acids. 


\section{$\underline{\text { References }}$}

1. Loenen, W. A. M., Dryden, D. T. F., Raleigh, E. A., Wilson, G. G. \& Murray, N. E. Highlights of the DNA cutters: a short history of the restriction enzymes. Nucleic Acids Research 42, 3-19 (2014).

2. Jinek, M. et al. A Programmable Dual-RNA-Guided DNA Endonuclease in Adaptive Bacterial Immunity. Science 337, 816-821 (2012).

3. Collias, D. \& Beisel, C. L. CRISPR technologies and the search for the PAM-free nuclease. Nature Communications 12, 555 (2021).

4. Wang, J.-W. et al. CRISPR/Cas9 nuclease cleavage combined with Gibson assembly for seamless cloning. BioTechniques 58, 161-170 (2015).

5. Walton, R. T., Christie, K. A., Whittaker, M. N. \& Kleinstiver, B. P. Unconstrained genome targeting with near-PAMless engineered CRISPR-Cas9 variants. Science 368, 290-296 (2020).

6. Jiang, W., Bikard, D., Cox, D., Zhang, F. \& Marraffini, L. A. RNA-guided editing of bacterial genomes using CRISPR-Cas systems. Nat Biotechnol 31, 233-239 (2013).

7. Kleinstiver, B. P. et al. Engineered CRISPR-Cas9 nucleases with altered PAM specificities. Nature 523, 481-485 (2015).

8. Swarts, D. C. et al. DNA-guided DNA interference by a prokaryotic Argonaute. Nature 507, 258-261 (2014).

9. Enghiad, B. \& Zhao, H. Programmable DNA-Guided Artificial Restriction Enzymes. ACS Synth. Biol. 6, 752-757 (2017).

10. Richter, M. F. et al. Phage-assisted evolution of an adenine base editor with improved Cas domain compatibility and activity. Nature Biotechnology 38, 883-891 (2020).

11. Anzalone, A. V. et al. Search-and-replace genome editing without double-strand breaks or donor DNA. Nature 576, 149-157 (2019).

12. Gibson, D. G. et al. Enzymatic assembly of DNA molecules up to several hundred kilobases. Nat Methods 6, 343-345 (2009).

13. Kleinstiver, B. P. et al. High-fidelity CRISPR-Cas9 nucleases with no detectable genome-wide off-target effects. Nature 529, 490-495 (2016).

14. Nishimasu, H. et al. Crystal Structure of Cas9 in Complex with Guide RNA and Target DNA. Cell 156, 935-949 (2014).

15. Anders, C., Niewoehner, O., Duerst, A. \& Jinek, M. Structural basis of PAM-dependent target DNA recognition by the Cas9 endonuclease. Nature 513, 569-573 (2014).

16. Chen, Z. \& Zhao, H. A highly sensitive selection method for directed evolution of homing endonucleases. Nucleic Acids Research 33, e154-e154 (2005).

17. Kleinstiver, B. P., Fernandes, A. D., Gloor, G. B. \& Edgell, D. R. A unified genetic, computational and experimental framework identifies functionally relevant residues of the homing endonuclease I-Bmol. Nucleic Acids Research 38, 2411-2427 (2010).

18. Zuo, Z. et al. Structural and functional insights into the bona fide catalytic state of Streptococcus pyogenes Cas9 HNH nuclease domain. eLife 8, e46500 (2019).

19. Hirano, S., Nishimasu, H., Ishitani, R. \& Nureki, O. Structural Basis for the Altered PAM Specificities of Engineered CRISPR-Cas9. Molecular Cell 61, 886-894 (2016).

20. Anders, C., Bargsten, K. \& Jinek, M. Structural Plasticity of PAM Recognition by Engineered Variants of the RNA-Guided Endonuclease Cas9. Molecular Cell 61, 895-902 (2016). 
21. Moreb, E. A., Hutmacher, M. \& Lynch, M. D. CRISPR-Cas "Non-Target" Sites Inhibit On-Target Cutting Rates. The CRISPR Journal 3, 550-561 (2020).

22. Moreb, E. A. \& Lynch, M. D. Genome dependent Cas9/gRNA search time underlies sequence dependent gRNA activity. Nat Commun 12, 5034 (2021).

23. Walton, R. T., Hsu, J. Y., Joung, J. K. \& Kleinstiver, B. P. Scalable characterization of the PAM requirements of CRISPR-Cas enzymes using HT-PAMDA. Nat Protoc 16, 1511-1547 (2021).

24. Gurevich, V. V., Pokrovskaya, I. D., Obukhova, T. A. \& Zozulya, S. A. Preparative in vitro mRNA synthesis using SP6 and T7 RNA polymerases. Analytical Biochemistry 195, 207-213 (1991).

25. Shams, A. et al. Comprehensive deletion landscape of CRISPR-Cas9 identifies minimal RNA-guided DNA-binding modules. Nat Commun 12, 5664 (2021).

26. $\mathrm{Gu}, \mathrm{W}$. et al. Depletion of Abundant Sequences by Hybridization (DASH): using Cas9 to remove unwanted high-abundance species in sequencing libraries and molecular counting applications. Genome Biology 17, 41 (2016).

27. Gilpatrick, T. et al. Targeted nanopore sequencing with Cas9-guided adapter ligation. Nat Biotechno/ 38, 433-438 (2020).

28. Rohland, N. \& Reich, D. Cost-effective, high-throughput DNA sequencing libraries for multiplexed target capture. Genome Res. 22, 939-946 (2012).

29. Karvelis, T. et al. Rapid characterization of CRISPR-Cas9 protospacer adjacent motif sequence elements. Genome Biology 16, 253 (2015).

30. Clement, K. et al. CRISPResso2 provides accurate and rapid genome editing sequence analysis. Nature Biotechnology 37, 224-226 (2019). 